Intuitionistic fuzzy theory and its application in economy, technology and management

\title{
MULTI-CRITERIA DECISION-MAKING METHOD BASED ON INTUITIONISTIC TRAPEZOIDAL FUZZY PRIORITISED OWA OPERATOR
}

\author{
Peide LIU', Ye LI ${ }^{\mathrm{b}, \mathrm{c}}$, Jurgita ANTUCHEVIČIENÉ ${ }^{\mathrm{d}}$ \\ ${ }^{a}$ School of Management Science and Engineering, Shandong University of Finance and Economics, \\ Jinan Shandong 250014, China \\ ${ }^{b}$ School of Economic and Management, Beijing Jiaotong University, Beijing 100044, China \\ ${ }^{c}$ Beijing Engineering Research Center of Green Building Technology, Beijing 100070, China \\ ${ }^{d}$ Department of Construction Technology and Management, \\ Vilnius Gediminas Technical University, LT-10223 Vilnius, Lithuania
}

Received 23 June 2015; accepted 8 December 2015

\begin{abstract}
In the real decision-making, there are many multiple attribute decision-making (MADM) problems, in which there exists the prioritised relationship among decision-making attributes. In this paper, with respect to the prioritised multi-criteria decision-making problems under intuitionistic trapezoidal fuzzy information, a new decision-making method on the basis of the intuitionistic trapezoidal fuzzy prioritised ordered weighted aggregation operator has been proposed. Firstly, the definitions, operational rules and characteristics of intuitionistic trapezoidal fuzzy numbers and POWA operator have been introduced. Then, intuitionistic trapezoidal fuzzy prioritised ordered weighted aggregation (ITFPOWA) operator has been defined as well as the computational method of associated weight, and some properties have been studied and proved. Furthermore, based on the ITFPOWA operator, an approach to the multi-criteria decision-making with intuitionistic trapezoidal fuzzy numbers has been established. Finally, an illustrative example has been given to prove the evaluation procedures of the developed approach and to demonstrate its practicality and validity.
\end{abstract}

Keywords: MADM, prioritised, intuitionistic trapezoidal fuzzy numbers, POWA, ITFPOWA.

JEL Classification: C44, C60.

\section{Introduction}

There are many multi-attribute decision-making (MADM) problems, which received considerable attention in the past few years. Since Zadeh (1965) proposed the theory of fuzzy set (FS), the research on fuzzy MADM (FMADM) problems has become a hot focus

Corresponding author Jurgita Antuchevičienè

E-mail: jurgita.antucheviciene@vgtu.lt 
(Merigó et al. 2015). Based on the fuzzy set, Atanassov (1986) presented the intuitionistic fuzzy set (IFS) by adding a new non-membership function, and then Atanassov and Gargov (1989), Atanassov (1994) proposed the interval-valued intuitionistic fuzzy set (IVIFS), which is a generalisation of the FS and IFS. The basic feature of the IVIFS is that the values of its membership function and non-membership function take the form of interval numbers rather than crisp numbers. On the basis of the presented theory, a great amount of extensions have been made (Yu, Shi 2015). Shu et al. (2006) presented the definition of intuitionistic triangular fuzzy number, and constructed an algorithm of the intuitionistic fuzzy fault-tree analysis. Zhang and Liu (2010) defined the triangular intuitionistic fuzzy numbers by extending the membership degree and the non-membership degree to the triangular fuzzy numbers, and proposed the weighted arithmetic averaging operator and the weighted geometric average operator. Furthermore, based on these operators, a method of multiple attribute group decision-making (MAGDM) with triangular intuitionistic fuzzy information has been established. New arithmetic operations and logic operators for triangular intuitionistic fuzzy numbers and a new method based on evidential reasoning were presented by Wang et al. (2013a, 2013b). The hesitant FMADM problem was investigated and some prioritised aggregation operators for aggregating hesitant fuzzy information were proposed, namely, hesitant fuzzy prioritised weighted average (HFPWA) operator and hesitant fuzzy prioritised weighted geometric (HFPWG) operator (Wei 2012). Some prioritised aggregation operators for aggregating triangular fuzzy information have been developed by Zhao et al. (2013). Fuzzy number intuitionistic fuzzy prioritised weighted average (FNIFPWA) operator and fuzzy number intuitionistic fuzzy prioritised weighted geometric (FNIFPWG) operator were proposed by Lin et al. (2013). The method with intuitionistic interval fuzzy information was applied to group decision-making (Wang et al. 2014).

At present, there are some studies on the intuitionistic trapezoidal fuzzy numbers mainly including the following: Wang (2008) proposed the conception of the intuitionistic trapezoidal fuzzy number and interval intuitionistic trapezoidal fuzzy number. Wang and Zhang (2008) proposed a programming method for the MADM problems with intuitionistic trapezoidal fuzzy number and incomplete weight information. Wan and Dong (2010) defined a new ranking method by using the coordinates of gravity centre about intuitionistic trapezoidal fuzzy number, and proposed the ordered weighted aggregation operator and hybrid aggregation operator to solve the MADM problems with intuitionistic trapezoidal fuzzy numbers. Wei (2010) proposed the intuitionistic trapezoidal fuzzy ordered weighted averaging (ITFOWA) operator and intuitionistic trapezoidal fuzzy hybrid aggregation (ITFHA) operator and pointed out a method with respect to MAGDM problems, where the attribute values are intuitionistic trapezoidal fuzzy information. The weighted geometric aggregation operator of trapezoidal fuzzy numbers for group decision-making was presented by Liu and Jin (2012a). Some new aggregation operators including interval intuitionistic trapezoidal fuzzy ordered weighted geometric (IITFOWG) operator and interval intuitionistic trapezoidal fuzzy hybrid geometric (IITFHG) operator were proposed, and some desirable properties of these operators were studied by Wei et al. (2012). Zhang et al. (2013) proposed a grey relational projection method for the MADM problems with the intuitionistic trapezoidal fuzzy number and the unknown weight information. Liu and $\mathrm{Yu}$ 
(2013) proposed the method of calculating density weighted vector and developed some density aggregation operators based on interval numbers and intuitionistic trapezoidal fuzzy numbers. Then, a multiple attribute decision-making method for the MADM problems with the intuitionistic trapezoidal fuzzy numbers was presented. Liu, D. P. and Liu, Y. (2014) proposed an intuitionistic trapezoidal fuzzy power generalised weighted average (ITFPGWA) operator, and propounded an approach to deal with group decision-making problems under intuitionistic trapezoidal fuzzy information based on the ITFPGWA operator. Chen and Liu (2014) proposed the intuitionistic trapezoidal fuzzy generalised Heronian OWA operator, and applied it to the MADM problems where the evaluation of information is depicted by intuitionistic trapezoidal fuzzy information. Heronian mean operators were applied for group decision-making (Liu et al. 2014). Uncertain linguistic variables and uncertain linguistic operators were analysed (Liu, Jin 2012b, 2012c; Liu, Yu 2014; Liu, Wang 2014).

The above researches were mainly on the MADM problems, in which attributes have been independent of each other and had no precedence relationship. However, in real decision-making, there exists a kind of MADM problems with the precedence relationship between the attributes. Yager (2004) studied this kind of the decision-making problems and emphasised that the importance of attributes with a lower priority has been determined on the basis of the extent, to which alternatives should satisfy the attributes with higher priority. Then, Yager (2008) proposed a prioritised aggregated operator and further constructed the prior "anding" operator and the prior "oring" operator in 2008. Based on prioritised aggregated operator, Yager (2009) proposed the prioritised ordered weighted average (OWA) operator for the prioritised decision-making problems, in which there exists a relationship between the criteria. Zeng et al. (2014) applied the weighted average operator to business decision-making. Yan et al. (2011) used the Hamacher parameterised t-norms to induce the priority weight for each priority level and proposed a benchmark-based approach to induce the priority weight for each priority level. Then, target-oriented decision analysis has been utilised to obtain the benchmark achievement for fuzzy requirements. Some of Hamaxer aggregation operators were applied to group decision-making (Liu 2014). Some new aggregation operators based on the Choquet integral and Einstein operations and considering the interactions phenomena among the decision-making criteria or their ordered positions were proposed by Xu et al. (2014). The intuitionistic fuzzy group decision-making problem, in which all the experts use the intuitionistic fuzzy preference relations (IFPRs) to express their preferences was analysed by Liao et al. (2015).

$\mathrm{Xu}$ et al. (2011) defined intuitionistic fuzzy prioritised ordered weighted average (IFPOWA) operator and established the corresponding method on the basis of IFPOWA operator. Guo et al. (2011) developed the prioritised ordered weighted C-OWA (POWCOWA) operator and prioritised ordered weighted C-OWG (POWC-OWG) operator, and analysed some of their characteristics. Based on POWC-OWA operator, a new method was constructed for solving the MADM problems, in which the attribute values are interval numbers.

Based on the aforementioned analysis, aiming at the MADM problems, in which there exists a priority relationship between the criteria and the criteria values are intuitionistic 
trapezoidal fuzzy numbers, we integrate intuitionistic trapezoidal fuzzy numbers and prioritised aggregated operator, and propose the intuitionistic trapezoidal fuzzy prioritised ordered weighted average (ITFPOWA) operator. Then, we propose an MADM method on the basis of the ITFPOWA operator. In the end, an illustrative example has been given to prove the effectiveness and feasibility of the method.

In order to do so, the remainder of this paper is organised as follows: in Section 1, we have briefly reviewed some basic concepts, operational rules, comparison method and the expected value of the intuitionistic trapezoidal fuzzy numbers, and introduced the definition of the POWA operator and the relevant weights. In Section 2, we have proposed an intuitionistic trapezoidal fuzzy prioritised ordered weighted aggregation (ITFPOWA) operator, and discussed some properties. Section 3 gives a method for the multi-criteria decision-making with intuitionistic trapezoidal fuzzy numbers based on the ITFPOWA operator. In Section 4, we have given an example to show the decision-making steps. The last Section ends this paper with some conclusions.

\section{Preliminaries}

\subsection{Intuitionistic trapezoidal fuzzy numbers}

Definition 1. Let $\tilde{a}$ be an intuitionistic trapezoidal fuzzy number, if its membership function is defined as (Wang, Zhang 2008):

$$
u_{\tilde{a}(x)}=\left\{\begin{array}{c}
\frac{x-a}{b-a} u_{\tilde{a}}, a \leq x<b ; \\
u_{\tilde{a}}, b \leq x \leq c ; \\
\frac{d-x}{d-c} u_{\tilde{a}}, c<x \leq d ; \\
0
\end{array}\right.
$$

and its non-membership function is defined as

$$
v_{\tilde{a}(x)}=\left\{\begin{array}{c}
\frac{b-x+v_{\tilde{a}}\left(x-a_{1}\right)}{b-a_{1}}, a_{1} \leq x<b ; \\
v_{\tilde{a}}, b \leq x \leq c ; \\
\frac{x-\epsilon v_{\tilde{a}}\left(d_{1} x\right)}{d_{1}-c}, c<x \leq d_{1} ; \\
0
\end{array}\right.
$$

where $0 \leq \mu_{\tilde{a}} \leq 1,0 \leq v_{\tilde{a}} \leq 1$ and $0 \leq \mu_{\tilde{a}}+v_{\tilde{a}} \leq 1 ; a, a_{1}, b, c, d, d_{1} \in R$. The intuitionistic trapezoidal fuzzy number is denoted as $\tilde{a}=\left\langle\left([a, b, c, d] ; \mu_{\tilde{a}}\right),\left(\left[a_{1}, b, c, d_{1}\right] ; v_{\tilde{a}}\right)\right\rangle$. Comparing with trapezoidal fuzzy numbers, the intuitionistic trapezoidal fuzzy numbers have another parameter: non-membership function, which is used to depict the degree of which the decision makers think that the element does not belong to $\left[a_{1}, b, c, d_{1}\right]$. When $\mu_{\tilde{a}}=1, v_{\tilde{a}}=0$, 
$\tilde{a}$ is reduced to a trapezoidal fuzzy number. When $b=c$, the intuitionistic trapezoidal fuzzy numbers become intuitionistic triangular fuzzy numbers. Generally, there is $[a, b, c, d]=\left[a_{1}, b, c, d_{1}\right]$ in intuitionistic trapezoidal fuzzy number $\tilde{a}$, and then we can express it as $\tilde{a}=<[a, b, c, d] ; \mu_{\tilde{a}}, v_{\tilde{a}}>. \pi_{\tilde{a}}=1-u_{\tilde{a}}-v_{\tilde{a}}$ indicates the hesitated degree of $\tilde{a}$. The smaller $\pi_{\tilde{a}}$ is, the more certain the fuzzy number is. If $d \geq c \geq b \geq a \geq 0$, we can call $\tilde{a}$ as positive intuitionistic trapezoidal fuzzy numbers.

Comparing with the intuitionistic fuzzy numbers, intuitionistic trapezoidal fuzzy numbers added to trapezoidal fuzzy numbers $[a, b, c, d]$ can express different dimensional decision-making information more exactly.

Definition 2 (Wang, Zhang 2008). Let $\tilde{a}_{1}=<\left[a_{1}, b_{1}, c_{1}, d_{1}\right] ; u_{\tilde{a}_{1}}, v_{\tilde{a}_{1}}>$ and $\tilde{a}_{2}=<\left[a_{2}, b_{2}, c_{2}, d_{2}\right] ; u_{\tilde{a}_{2}}, v_{\tilde{a}_{2}}>$ be two positive intuitionistic trapezoidal fuzzy numbers, $\lambda \geq 0$, then the operational laws between $\tilde{a}_{1}$ and $\tilde{a}_{2}$ can be defined as follow:

$$
\begin{aligned}
& \tilde{a}_{1}+\tilde{a}_{2}=<\left[a_{1}+a_{2}, b_{1}+b_{2}, c_{1}+c_{2}, d_{1}+d_{2}\right] ; u_{\tilde{a}_{1}}+u_{\tilde{a}_{2}}-u_{\tilde{a}_{1}} u_{\tilde{a}_{2}}, v_{\tilde{a}_{1}} v_{\tilde{a}_{2}}>; \\
& \tilde{a}_{1} \tilde{a}_{2}=<\left[a_{1} a_{2}, b_{1} b_{2}, c_{1} c_{2}, d_{1} d_{2}\right] ; u_{\tilde{a}_{1}} u_{\tilde{a}_{2}}, v_{\tilde{a}_{1}} v_{\tilde{a}_{2}}-v_{\tilde{a}_{1}} v_{\tilde{a}_{2}}>; \\
& \lambda \tilde{a}_{1}=<\left[\lambda a_{1}, \lambda b_{1}, \lambda c_{1}, \lambda d_{1}\right] ; 1-\left(1-u_{\tilde{a}_{1}}\right)^{\lambda}, v_{\tilde{a}_{1}}^{\lambda}>; \\
& \tilde{a}_{1}^{\lambda}=<\left[a_{1}^{\lambda}, b_{1}^{\lambda}, c_{1}^{\lambda}, d_{1}^{\lambda}\right] ; u_{\tilde{a}_{1}}^{\lambda}, 1-\left(1-v_{\tilde{a}_{1}}\right)^{\lambda}>.
\end{aligned}
$$

Theorem 1. Let $\tilde{a}_{1}=<\left[a_{1}, b_{1}, c_{1}, d_{1}\right] ; \mu_{\tilde{a}_{1}}, v_{\tilde{a}_{1}}>$ and $\tilde{a}_{2}=<\left[a_{2}, b_{2}, c_{2}, d_{2}\right] ; \mu_{\tilde{a}_{2}}, v_{\tilde{a}_{2}}>$ be two positive intuitionistic trapezoidal fuzzy numbers, then there are the following properties about the calculation rules between $\tilde{a}_{1}$ and $\tilde{a}_{2}$ :

$$
\begin{aligned}
& \tilde{a}_{1}+\tilde{a}_{2}=\tilde{a}_{2}+\tilde{a}_{1} ; \\
& \tilde{a}_{1} \tilde{a}_{2}=\tilde{a}_{2} \tilde{a}_{1} ; \\
& \lambda\left(\tilde{a}_{1}+\tilde{a}_{2}\right)=\lambda \tilde{a}_{1}+\lambda \tilde{a}_{2}, \lambda \geq 0 ; \\
& \lambda_{1} \tilde{a}_{1}+\lambda_{2} \tilde{a}_{1}=\left(\lambda_{1}+\lambda_{2}\right) \tilde{a}_{1}, \lambda_{1}, \lambda_{2} \geq 0 ; \\
& \tilde{a}_{1}^{\lambda_{1}} \tilde{a}_{1}^{\lambda_{2}}=\left(\tilde{a}_{1}\right)^{\lambda_{1}+\lambda_{2}}, \lambda_{1}, \lambda_{2} \geq 0 ; \\
& \tilde{a}_{1}^{\lambda_{1}} \tilde{a}_{2} \lambda_{1}=\left(\tilde{a}_{1} \tilde{a}_{2}\right)^{\lambda_{1}}, \lambda_{1} \geq 0 .
\end{aligned}
$$

It is easy to prove Theorem 1; therefore, it is omitted here.

Definition 3. Let $\tilde{a}_{1}=<\left[a_{1}, b_{1}, c_{1}, d_{1}\right] ; u_{\tilde{a}_{1}}, v_{\tilde{a}_{1}}>$ and $\tilde{a}_{2}=<\left[a_{2}, b_{2}, c_{2}, d_{2}\right] ; u_{\tilde{a}_{2}}, v_{\tilde{a}_{2}}>$ be two positive intuitionistic trapezoidal fuzzy numbers, then the possibility degree of intuitionistic trapezoidal fuzzy numbers $\tilde{a}_{1} \geq \tilde{a}_{2}$ is:

$$
p\left(\tilde{a}_{1} \geq \tilde{a}_{2}\right)=\left(1+\frac{\left(\eta_{\tilde{a}_{1}} a_{1}-\eta_{\tilde{a}_{2}} a_{2}\right)+\left(\eta_{\tilde{a}_{1}} b_{1}-\eta_{\tilde{a}_{2}} b_{2}\right)+\left(\eta_{\tilde{a}_{1}} c_{1}-\eta_{\tilde{a}_{2}} c_{2}\right)+\left(\eta_{\tilde{a}_{1}} d_{1}-\eta_{\tilde{a}_{2}} d_{2}\right)}{\left|\eta_{\tilde{a}_{1}} a_{1}-\eta_{\tilde{a}_{2}} a_{2}\right|+\left|\eta_{\tilde{a}_{1}} b_{1}-\eta_{\tilde{a}_{2}} b_{2}\right|+\left|\eta_{\tilde{a}_{1}} c_{1}-\eta_{\tilde{a}_{2}} c_{2}\right|+\left|\eta_{\tilde{a}_{1}} d_{1}-\eta_{\tilde{a}_{2}} d_{2}\right|}\right) / 2
$$

where $\eta_{\tilde{a}_{1}}=1+u_{\tilde{a}_{1}}-v_{\tilde{a}_{1}}, \eta_{\tilde{a}_{2}}=1+u_{\tilde{a}_{2}}-v_{\tilde{a}_{2}}$.

Suppose $p\left(\tilde{a}_{1} \geq \tilde{a}_{2}\right)=0.5$, when $\tilde{a}_{1}=\tilde{a}_{2}$, i.e. $a_{1}=a_{2}, b_{1}=b_{2}, c_{1}=c_{2}, d_{1}=d_{2}, u_{\tilde{a}_{1}}=$ $u_{\tilde{a}_{2}}, v_{\tilde{a}_{1}}=v_{\tilde{a}_{2}} \cdot$ 
For a set of intuitionistic trapezoidal fuzzy numbers $\tilde{a}_{i}=<\left[a_{i}, b_{i}, c_{i}, d_{i}\right] ; u_{\tilde{a}_{i}}, v_{\tilde{a}_{i}}>,(i=$ $1,2, \ldots, n)$, the possibility degree $p_{i j}=p\left(\tilde{a}_{i}>\tilde{a}_{j}\right),(i=1,2, \ldots, n ; j=1,2, \ldots, m)$ can be obtained by the pair-wise comparison, then the possibility degree matrix $P=\left(p_{i j \times n}\right)$ was built. Let $\lambda_{i}=\sum_{j=1}^{n} p_{i j}$, we can get the ranking vector $\lambda=\left(\lambda_{1}, \lambda_{2}, \ldots, \lambda_{n}\right)$. The bigger $\lambda_{i}$ is, the bigger the intuitionistic trapezoidal fuzzy number is.

Definition 4. For the positive intuitionistic trapezoidal fuzzy number $\tilde{a}=\left\langle[a, b, c, d] ; \mu_{\tilde{a}}, v_{\tilde{a}}\right\rangle$, its expected value is shown as follows:

$$
I(\tilde{a})=\frac{1}{8} \times\left[(a+b+c+d) \times\left(1+\mu_{\tilde{a}}-v_{\tilde{a}}\right)\right],
$$

where $(a+b+c+d) / 4$ is the expectation of trapezoidal fuzzy number $[a, b, c, d]$, $\left(u_{\tilde{a}}+1-v_{\tilde{a}}\right) / 2$ is the expectation of intuitionistic fuzzy number $\left(\mu_{\tilde{a}}, v_{\tilde{a}}\right)$.

\subsection{Prioritised ordered weighted aggregated operator (POWA)}

\subsubsection{Definition of a POWA operator}

Definition 5 (Yager 2009). Suppose that we have a collection of criteria partitioned into $p$ distinct categories $H_{1}, H_{2}, \ldots, H_{p}$ such that $H_{i}=\left\{a_{i 1}, a_{i 2}, \ldots, a_{i n_{i}}\right\}$. Here $a_{i j}$ are the criteria in the category $H_{i}$. We assume a prioritisation between these categories $H_{1}>H_{2}>\ldots>H_{p}$.

The criteria in the class $H_{i}$ have a higher priority than those in $H_{k}$ if $i<k$. The total set of criteria is $C=\bigcup_{i=1}^{p} H_{i}$. We assume $n=\sum_{i=1}^{p} n_{i}$ is the total number of criteria. If
$f_{p}:[0,1]^{n} \rightarrow[0,1]$, then

$$
f_{p}\left(a_{1}, a_{2}, \ldots, a_{n}\right)=\sum_{i=1}^{p} w_{i} f_{i}
$$

where $f_{i}=\sum^{n_{i}} \omega_{i j} a_{i j}$ is the aggregated value in the category $H_{i}$ by weighted average operator $=\omega_{i}=\left(\omega_{i 1}, \omega_{i 2}, \ldots, \omega_{i n_{i}}\right)^{T}$ is the attribute weight vector of $a_{i j}\left(j=1,2, \ldots, n_{i}\right)$. Here, $0 \leq \omega_{i j} \leq 1, \sum_{i=1}^{p} \sum_{j=1}^{n_{i}} \omega_{i j}=1$. Suppose $\tau=\left(\tau_{1}, \tau_{2}, \ldots, \tau_{p}\right)$ is the position weight of $f_{i}(i=1,2, \ldots, p)$, calculated in the non-priority conditions, such that $0 \leq \tau_{i} \leq 1, \sum_{i=1}^{p} \tau_{i}=1$ and $w=\left(w_{1}, w_{2}, \ldots, w_{p}\right)_{p}^{T}$ is the weight vector associated with the category $H_{1}, H_{2}, \ldots, H_{p}$, $w_{i} \in[0,1], i=1,2, \ldots, p, \sum_{i=1}^{p} w_{i}=1$, then function $f_{p}$ is called as POWA operator.

\subsubsection{Position weight}

About the position weight $\tau=\left(\tau_{1}, \tau_{2}, \ldots, \tau_{p}\right)$, it can be determined according to the actual needs, or according to the following methods.

Firstly, we need to set the value $\alpha \in[0,1]$ as expected attitude characteristics of the weighted vector. So, we calculate the position weighted vector $\tau=\left(\tau_{1}, \tau_{2}, \ldots, \tau_{p}\right)$ used by the following math formula (O'Hagan 1990): 


$$
\begin{array}{cl}
\text { Max } & -\sum_{j=1}^{p} \tau_{j} \ln \left(\tau_{j}\right) \\
\mathrm{S} / \mathrm{t} \quad & \sum_{j=1}^{p} \tau_{j} \frac{p-j}{p-1}=\alpha \\
& \sum_{j=1}^{p} \tau_{j}=1 \\
& \leq \leq \tau_{j} \leq 1 .
\end{array}
$$

In addition, the position weight vector $\tau$ can be determined by combinatorial numbers, as follows (Wang, $\mathrm{Xu} 2008$ ):

$$
\tau_{i+1}=\frac{C_{p-1}^{i}}{2^{p-1}}, \quad i=0,1, \cdots, p-1
$$

1.2.3. Weight vector associated with the category $H_{1}, H_{2}, \ldots, H_{p}$ (Yager 2004, 2008)

Suppose $T_{i}$ is the measurement of the relative importance in every prioritised level. Let $T_{1}=1, V_{i}=f_{i}$, then

$$
T_{i}=\prod_{k=1}^{i-1} V_{k}=T_{i-1} V_{k-1}
$$

Let $r_{i}$ be a normalised priority-based weight, and $r_{\sigma(i)}$ be the corresponding prioritised weight of $f_{\sigma(i)}, f_{\sigma(i)}$ is the ith largest element of $f_{i}, i=1,2, \ldots, p$, and let $R_{i}=\sum_{i=1}^{p} r_{\sigma(i)}$ We
have:

Choose the BUM function:

$$
r_{i}=\frac{T_{i}}{\sum_{i=1}^{p} T_{i}}, \quad i=1,2, \ldots, p
$$

$$
h(z)=\sum_{i=1}^{j-1} \tau_{i}+\tau_{j}(p z-(j-1)),(j-1) / p \leq z \leq j / p .
$$

Let the weight vector associated with the category $\left(H_{1}, H_{2}, \ldots, H_{p}\right)$ be $w=\left(w_{1}, w_{2}, \ldots, w_{p}\right)^{T}$ and $h\left(R_{0}\right)=0$. Then,

$$
w_{i}=h\left(R_{j+1}\right)-h\left(R_{j}\right)
$$

\section{Intuitionistic trapezoidal fuzzy prioritised ordered weighted aggregation (ITFPOWA) operator}

\subsection{Definition of ITFPOWA operator}

Supposed there is a set of attributes $C=\left(C_{11}, C_{12}, \ldots, C_{1 n_{1}}, C_{21}, C_{22}, \ldots, C_{2 n_{2}}, C_{p 1}, C_{p 2}, \ldots, C_{p n_{p}}\right)$ and $H_{i}$ is the corresponding prioritised degree, and meets $H_{1}>H_{2}>\ldots>H_{p}$ so that $H_{i}=\left\{\tilde{a}_{i 1}, \tilde{a}_{i 2}, \ldots, \tilde{a}_{i n_{i}}\right\}, \tilde{a}_{i j}$ are the criteria values in the category $H_{i}$ under the attribute $C_{i j}$, and $\tilde{a}_{i j}=<\left[a_{i j}^{a}, a_{i j}^{b}, a_{i j}^{c}, a_{i j}^{d}\right] ; u_{i j}, v_{i j}>, 0 \leq a_{i j}^{a}, a_{i j}^{b}, a_{i j}^{c}, a_{i j}^{d} \leq 1,0 \leq u_{i j} \leq 1 ; 0 \leq v_{i j} \leq 1,0 \leq u_{i j}+v_{i j} \leq 1$, 
$i=1,2, \ldots, p, j=1,2, \ldots, n_{i},$. where $n=\sum_{i=1}^{p} n_{i} . \omega_{i}=\left(\omega_{i 1}, \omega_{i 2}, \ldots, \omega_{i n_{i}}\right)^{T}$ is the attribute weight of $C_{i j}, i=1,2, \ldots, p, j=1,2, \ldots, n_{i}, 0 \leq \omega_{i j} \leq 1, \sum_{i=1}^{p} \sum_{j=1}^{n_{i}} \omega_{i j}=1 . \tilde{f}=\left(\tilde{f}_{1}, \tilde{f}_{2}, \ldots, \tilde{f}_{p}\right)$ is the aggregated vector in the category $H_{i}(i=1,2, \ldots, p)$ by intuitionistic trapezoidal fuzzy weighted average operator, where

$$
\begin{aligned}
& \tilde{f}_{i}=<\left[f_{i}^{a}, f_{i}^{b}, f_{i}^{c}, f_{i}^{d}\right], f u_{i}, f v_{i}>=\sum_{j=1}^{n_{i}} \omega_{i j} \tilde{a}_{i j}= \\
& <\left[\sum_{j=1}^{n_{i}} \omega_{i j} a_{i j}^{a}, \sum_{j=1}^{n_{i}} \omega_{i j} a_{i j}^{b}, \sum_{j=1}^{n_{i}} \omega_{i j} a_{i j}^{c}, \sum_{j=1}^{n_{i}} \omega_{i j} a_{i j}^{d}\right] ; 1-\prod_{j=1}^{n_{i}}\left(1-u_{i j}\right)^{\omega_{i j}}, \prod_{j=1}^{n_{i}} v_{i j}^{\omega_{i j}}>.
\end{aligned}
$$

Definition 6. If $\tilde{F}:[0,1]^{n} \rightarrow[0,1]$, make:

$$
\tilde{F}=\sum_{i=1}^{p} w_{i} \tilde{f}_{i}=\left\langle\left[\sum_{i=1}^{p} w_{i} f_{i}^{a}, \sum_{i=1}^{p} w_{i} f_{i}^{b}, \sum_{i=1}^{p} w_{i} f_{i}^{c}, \sum_{i=1}^{p} w_{i} f_{i}^{d}\right] ; 1-\prod_{i=1}^{p}\left(1-f u_{i}\right)^{w_{i}}, \prod_{i=1}^{p} f v_{i}^{w_{i}}\right\rangle,
$$

where $w=\left(w_{1}, w_{2}, \ldots, w_{p}\right)^{T}$ is the associated weighted vector to the function $\tilde{F}$, $0 \leq w_{i} \leq 1, \sum_{i=1}^{p} w_{i}=1$; then, we call the function $\tilde{F}$ an intuitionistic trapezoidal fuzzy prioritised ordered weighted aggregated operator, referred to as the ITFPOWA operator.

\subsection{Associated weighted vector}

Suppose $T_{i}$ is the measurement of the relative importance of intuitionistic trapezoidal fuzzy numbers in every prioritised level, let $T_{1}=1, V_{i}$ is the expected value of the intuitionistic trapezoidal fuzzy number $\tilde{f}_{i}$. Then,

$$
T_{i}=\prod_{k=1}^{i-1} V_{k}=T_{i-1} V_{k-1}
$$

Let $r_{i}$ be the normalised prioritised weight, $r_{\sigma(i)}$ - the corresponding prioritised weight of $\tilde{f}_{\sigma(i)}, \tilde{f}_{\sigma(i)}$ - the $i$ th largest element of $\left(\tilde{f}_{1}, \tilde{f}_{2}, \ldots, \tilde{f}_{p}\right)$, and let $R_{i}=\sum_{i=1}^{p} r_{\sigma(i)}$. We have:

Choose the BUM function:

$$
r_{i}=\frac{T_{i}}{\sum_{i=1}^{p} T_{i}}, i=1,2, \ldots, p
$$

$$
h(z)=\sum_{i=1}^{j-1} \tau_{i}+\tau_{j}(p z-(j-1))(j-1) / p \leq z \leq j / p .
$$

Let the weight vector associated with the category $H_{1}, H_{2}, \ldots, H_{p}$ be $w=\left(w_{1}, w_{2}, \ldots, w_{p}\right)^{T}$ and $h\left(R_{0}\right)=0$. Then,

$$
w_{i}=h\left(R_{j+1}\right)-h\left(R_{j}\right) .
$$




\subsection{Characteristic of the ITFPOWA operator}

Theorem 2. Let $\tilde{a}_{i}=<\left[a_{i}^{a}, a_{i}^{b}, a_{i}^{c}, a_{i}^{d}\right] ; u_{\tilde{a}_{i}}, v_{\tilde{a}_{i}}>(i=1,2, \ldots, n)$ be a set of intuitionistic trapezoidal fuzzy numbers, then the integrated value by ITFPOWA operator is still an intuitionistic trapezoidal fuzzy number, and

$$
\tilde{F}=<\left[\sum_{i=1}^{n} w_{i} a_{\sigma(i)}^{a}, \sum_{i=1}^{n} w_{i} a_{\sigma(i)}^{b}, \sum_{i=1}^{n} w_{i} a_{\sigma(i)}^{c}, \sum_{i=1}^{n} w_{i} a_{\sigma(i)}^{d}\right] ; 1-\prod_{i=1}^{n}\left(1-u_{\sigma(i)}\right)^{w_{i}}, \prod_{i=1}^{n} v_{\sigma(i)}^{w_{i}}>.
$$

Proof. We can use the mathematical induction method to prove this theorem. When $n=2$,

$$
\tilde{F}=w_{1} \tilde{a}_{\sigma(1)}+w_{2} \tilde{a}_{\sigma(2)},
$$

where $\tilde{a}_{\sigma(1)}=\max \left(\tilde{a}_{1}, \tilde{a}_{2}\right)$ and $\tilde{a}_{\sigma(2)}=\min \left(\tilde{a}_{1}, \tilde{a}_{2}\right)$ are both intuitionistic trapezoidal fuzzy numbers, $w_{1}, w_{2} \in[0,1]$ are both real numbers. From basic operations of intuitionistic trapezoidal fuzzy numbers, we can know, $\tilde{F}\left(\tilde{a}_{1}, \tilde{a}_{2}\right)$ is intuitionistic trapezoidal fuzzy number, and

$$
\begin{aligned}
& \tilde{F}=w_{1} \tilde{a}_{\sigma(1)}+w_{2} \tilde{a}_{\sigma(2)}= \\
& <\left[w_{1} a_{\sigma(1)}^{a}+w_{2} a_{\sigma(2)}^{a}, w_{1} a_{\sigma(1)}^{b}+w_{2} a_{\sigma(2)}^{b}, w_{1} a_{\sigma(1)}^{c}+w_{2} a_{\sigma(2)}^{c}, w_{1} a_{\sigma(1)}^{d}+w_{2} a_{\sigma(2)}^{d}\right], \\
& u_{\sigma(1)}+u_{\sigma(2)}-u_{\sigma(1)} u_{\sigma(2)}, v_{\sigma(1)} v_{\sigma(2)}>.
\end{aligned}
$$

Supposed $n=k$, theorem 2 is right, that is

$$
\begin{aligned}
& \tilde{F}=\sum_{i=1}^{k} w_{i} \tilde{a}_{\sigma(i)}= \\
& <\left[\sum_{i=1}^{k} w_{i} a_{\sigma(i)}^{a}, \sum_{i=1}^{k} w_{i} a_{\sigma(i)}^{b}, \sum_{i=1}^{k} w_{i} a_{\sigma(i)}^{c}, \sum_{i=1}^{k} w_{i} a_{\sigma(i)}^{d}\right] ; 1-\prod_{i=1}^{k}\left(1-u_{\sigma(i)}\right)^{w_{i}}, \prod_{i=1}^{k} v_{\sigma(i)}^{w_{i}}>.
\end{aligned}
$$

Then when $n=k+1$, we can get

$$
\begin{aligned}
& \tilde{F}=\sum_{i=1}^{k} w_{i} \tilde{a}_{\sigma(i)}+w_{k+1} \tilde{a}_{k+1}= \\
& <\left[\sum_{i=1}^{k+1} w_{i} a_{\sigma(i)}^{a}, \sum_{i=1}^{k+1} w_{i} a_{\sigma(i)}^{b}, \sum_{i=1}^{k+1} w_{i} a_{\sigma(i)}^{c}, \sum_{i=1}^{k+1} w_{i} a_{\sigma(i)}^{d}\right] ; 1-\prod_{i=1}^{k+1}\left(1-u_{\sigma(i)}\right)^{w_{i}}, \prod_{i=1}^{k+1} v_{\sigma(i)}^{w_{i}}>.
\end{aligned}
$$

So, when $n=k+1$, theorem 2 is still right.

According to the principle of mathematical induction method, we can state that Theorem 2 holds.

Theorem 3 (Idempotency). Let $\tilde{a}_{i}=<\left[a_{i}, b_{i}, c_{i}, d_{i}\right] ; u_{\tilde{a}_{i}}, v_{\tilde{a}_{i}}>(i=1,2, \ldots, n)$ be a set of intuitionistic trapezoidal fuzzy numbers, if the value of all intuitionistic trapezoidal fuzzy numbers is equal, that is $\tilde{a}_{i}=<\left[a_{i}, b_{i}, c_{i}, d_{i}\right] ; u_{\tilde{a}_{i}}, v_{\tilde{a}_{i}}>=\tilde{a}=<[a, b, c, d] ; u_{\tilde{a}}, v_{\tilde{a}}>$, then we have

$$
\tilde{F}=\tilde{a}=<[a, b, c, d] ; u_{\tilde{a}}, v_{\tilde{a}}>\text {. }
$$




\section{Proof:}

$$
\begin{aligned}
& \tilde{F}=\sum_{i=1}^{n} w_{i} \tilde{a}_{\sigma(i)}= \\
& <\left[\sum_{i=1}^{n} w_{i} a_{\sigma(i)}, \sum_{i=1}^{n} w_{i} b_{\sigma(i)}, \sum_{i=1}^{n} w_{i} c_{\sigma(i)}, \sum_{i=1}^{n} w_{i} d_{\sigma(i)}\right] ; 1-\prod_{i=1}^{n}\left(1-u_{\sigma(i)}\right)^{w_{i}}, \prod_{i=1}^{n} v_{\sigma(i)}^{w_{i}}>= \\
& <\left[\sum_{i=1}^{n} w_{i} a, \sum_{i=1}^{n} w_{i} b, \sum_{i=1}^{n} w_{i} b, \sum_{i=1}^{n} w_{i} d\right] ; 1-\prod_{i=1}^{n}(1-u)^{w_{i}}, \prod_{i=1}^{n} v^{w_{i}}>,
\end{aligned}
$$

because

$$
\begin{aligned}
& \sum_{i=1}^{n} w_{i} a=a, \sum_{i=1}^{n} w_{i} b=b, \sum_{i=1}^{n} w_{i} c=c, \sum_{i=1}^{n} w_{i} d=d ; \\
& 1-\prod_{i=1}^{n}(1-u)^{w_{i}}=1-(1-u)_{i=1}^{n} w_{i}=1-(1-u)=u, \\
& \prod_{i=1}^{n} v^{w_{i}}=v^{\sum_{i=1}^{n} w_{i}}=v .
\end{aligned}
$$

So, $\tilde{F}=\tilde{a}=<[a, b, c, d] ; u_{\tilde{a}}, v_{\tilde{a}}>$.

Theorem 4 (Boundedness). Let $\left.\tilde{a}_{i}=<\left[a_{i}, b_{i}, c_{i}, d_{i}\right] ; u_{\tilde{a}_{i}}, v_{\tilde{a}_{i}}\right)(i=1,2, \ldots, n>$ be a set of intuitionistic trapezoidal fuzzy numbers, and $\tilde{a}^{-}=\min _{i}<\left[a_{i}, b_{i}, c_{i}, d_{i}\right] ; u_{\tilde{a}_{i}}, v_{\tilde{a}_{i}}>$, $\tilde{a}^{+}=\max _{i}<\left[a_{i}, b_{i}, c_{i}, d_{i}\right] ; u_{\tilde{a}_{i}}, v_{\tilde{a}_{i}}>$, then $\tilde{a}^{-} \leq \tilde{F} \leq \tilde{a}^{+}$.

\section{Proof:}

Because $\tilde{a}_{\sigma(i)}$ is the $i$ th largest element in $\tilde{a}_{i}(i=1,2, \ldots, n)$, for any $i$, there is $\min \left(\tilde{a}_{\sigma(i)}\right) \leq \tilde{a}_{\sigma(i)} \leq \max \left(\tilde{a}_{\sigma(i)}\right)$. Then, there is :

$$
\sum_{i=1}^{n} w_{i} \tilde{a}_{\sigma(i)} \geq \sum_{i=1}^{n} w_{i} \min \left(\tilde{a}_{\sigma(i)}\right)=\min \left(\tilde{a}_{\sigma(i)}\right), \sum_{i=1}^{n} w_{i} \tilde{a}_{\sigma(i)} \leq \sum_{i=1}^{n} w_{i} \max \left(\tilde{a}_{\sigma(i)}\right)=\max \left(\tilde{a}_{\sigma(i)}\right) .
$$

So, we can get $\min \left(\tilde{a}_{\sigma(i)}\right) \leq \sum_{i=1}^{n} w_{i} \tilde{a}_{\sigma(i)} \leq \max \left(\tilde{a}_{\sigma(i)}\right)$, i.e., $\tilde{a}^{-} \leq \tilde{F} \leq \tilde{a}^{+}$.

\section{Multi-attribute decision-making method based on the ITFPOWA operator}

Let $A=\left\{A_{1}, A_{2}, \cdots, A_{m}\right\}$ be a discrete set of alternatives, and $C=\left(C_{11}, C_{12}, \ldots, C_{1 n_{1}}, C_{21}, C_{22}, \ldots\right.$, $\left.C_{2 n_{2}}, C_{p 1}, C_{p 2}, \ldots, C_{p n_{p}}\right)$ be the set of attributes, $H_{k}$ - the corresponding prioritised degree and meets $H_{1}>H_{2}>\ldots>H_{p}$ so that $H_{k}=\left\{\tilde{a}_{k 1}^{i}, \tilde{a}_{k 2}^{i}, \ldots, \tilde{a}_{k n_{k}}^{i}\right\}, \tilde{a}_{k j}^{i}$ - the criteria values in the category $H_{k}$ under the attribute $C_{k j}$ with respect to the alternative $A_{i}$, and $\tilde{a}_{k j}^{i}=<\left[a_{k j}^{i}, b_{k j}^{i}, b_{k j}^{i}, d_{k j}^{i}\right] ; u_{k j}^{i}, v_{k j}^{i}>, 0 \leq a_{k j}^{i}, b_{k j}^{i}, b_{k j}^{i}, d_{k j}^{i} \leq 1,0 \leq u_{k j}^{i} \leq 1 ; 0 \leq v_{k j}^{i} \leq 1,0 \leq u_{k j}^{i}+v_{k j}^{i} \leq 1$, $i=1,2, \ldots, m, k=1,2, \ldots, p, j=1,2, \ldots, n_{k}$. where $n=\sum_{k=1}^{p} n_{k}$.

$\omega=\left(\omega_{11}, \omega_{12}, \ldots, \omega_{1 n_{1}}, \omega_{21}, \omega_{22}, \ldots, \omega_{2 n_{2}}, \omega_{p 1}, \omega_{p 2}, \ldots, \omega_{p n_{p}}\right)^{T}$ is the attribute weight vector of $C_{k j}, k=1,2, \ldots, p, j=1,2, \ldots, n_{k}, 0 \leq \omega_{k j} \leq 1, \sum_{k=1}^{p} \sum_{j=1}^{n_{k}} \omega_{k j}=1 . \tilde{f}^{i}=\left(\tilde{f}_{1}^{i}, \tilde{f}_{2}^{i}, \ldots, \tilde{f}_{p}^{i}\right)$ is the aggregat- 
ed vector in the category $H_{k}(k=1,2, \ldots, p)$ with respect to the alternative $A_{i}$ by the intuitionistic trapezoidal fuzzy weighted average operator, $\tau=\left(\tau_{1}, \tau_{2}, \ldots, \tau_{p}\right)$ is the position weight of $\tilde{f}^{i}=\left(\tilde{f}_{1}^{i}, \tilde{f}_{2}^{i}, \ldots, \tilde{f}_{p}^{i}\right)$ calculated in the non-priority conditions, so that $0 \leq \tau_{k} \leq 1, \sum_{k=1}^{p} \tau_{k}=1$, and $w_{i}=\left(w_{i 1}, w_{i 2}, \ldots, w_{i p}\right)^{T}$ is the weight vector associated with the category $H_{1}, H_{2}, \ldots, H_{p}$ with respect to the alternative $A_{i}, w_{i k} \in[0,1], i=1,2, \ldots, m, k=1,2, \ldots, p, \sum_{k=1}^{p} w_{i k}=1$. Then, the
ranking of alternatives is required.

Next, we apply the ITFPOWA operator to MADM problems based on the intuitionistic trapezoidal fuzzy information.

Step 1. Get the aggregated information $\tilde{f}_{k}^{i}$ in the same category $H_{k}$ with respect to the alternative $A_{i}$.

We can use the intuitionistic trapezoidal fuzzy weighted average operator to integrate the information $\left(\tilde{a}_{k 1}^{i}, \tilde{a}_{k 2}^{i}, \ldots, \tilde{a}_{k n_{k}}^{i}\right)$ in the same category $H_{k}$, and get

$$
\tilde{f}_{k}^{i}=\sum_{j=1}^{n_{k}} \omega_{k j} \tilde{a}_{k j}^{i}
$$

Step 2. Calculate the position weight $\tau=\left(\tau_{1}, \tau_{2}, \ldots, \tau_{p}\right)$ in the non-priority conditions by Formulas (16) or (17).

Step 3. Calculate associated weighted vector $w_{i}=\left(w_{i 1}, w_{i 2}, \ldots, w_{i p}\right)^{T}$ according to Formulas (24-27).

Step 4. Calculate the integrated value $\tilde{f}^{i}$ by the ITFPOWA operator.

We can get

$$
\tilde{f}^{i}=\sum_{k=1}^{p} w_{i k} \tilde{f}_{k}^{i}
$$

Step 5. Rank the alternatives $A_{1}, A_{2}, \cdots, A_{m}$ by the matrix of possibility degree.

Step 6. Finished.

\section{Illustrative example}

\subsection{Problem formulation}

Consider the recruitment of human resource managers.

There are 4 recruiters (evaluation objects), $A=\left(A_{1}, A_{2}, A_{3}, A_{4}\right)$, and 8 evaluation indicators, $C=\left(C_{11}, C_{12}, C_{13}, C_{21}, C_{31}, C_{41}, C_{42}, C_{51}\right)$.

$H_{k}$ is the corresponding prioritised level, and has prioritised relationship $H_{1}>H_{2}>H_{3}>H_{4}>H_{5} . H_{1}$ (Ability level): Learning ability $C_{11}$, Management innovation ability $\mathrm{C}_{12}$, Communication and coordination ability $\mathrm{C}_{13} ; \mathrm{H}_{2}$ : Professional quality $\mathrm{C}_{21}$; $\mathrm{H}_{3}$ : Moral character $\mathrm{C}_{31} ; \mathrm{H}_{4}$ (Knowledge level): Professional knowledge $\mathrm{C}_{41}$, Laws and regulations knowledge $\mathrm{C}_{42} ; \mathrm{H}_{5}$ : State of health $\mathrm{C}_{51}$.

$\omega_{k}=\left(\omega_{k 1}, \omega_{k 2}, \ldots, \omega_{k n_{k}}\right)^{T}$ is the attribute weight vector, $0 \leq \omega_{k j} \leq 1, \sum_{k=1}^{p} \sum_{j=1}^{n_{k}} \omega_{k j}=1$.
$=(0.1,0.1,0.1)^{T}, \omega_{2}=0.2, \omega_{3}=0.2, \omega_{4}=(0.1,0.1)^{T}, \omega_{5}=0.1$. 
$\tilde{a}_{k j}^{i}$ is the criteria value in the category $H_{k}$ under the attribute $C_{k j}$ with respect to the alternative $A_{i}$, and $\tilde{a}_{k j}^{i}=<\left[a_{k j}^{i}, b_{k j}^{i}, b_{k j}^{i}, d_{k j}^{i}\right] ; u_{k j}^{i}, v_{k j}^{i}>, 0 \leq a_{k j}^{i}, b_{k j}^{i}, b_{k j}^{i}, d_{k j}^{i} \leq 1,0 \leq u_{k j}^{i} \leq 1 ; 0 \leq v_{k j}^{i} \leq 1$, $0 \leq u_{k j}^{i}+v_{k j}^{i} \leq 1, i=1,2,3,4, k=1,2,3,4,5, j=1,2, \ldots, n_{k}$.

Decision-making information is shown in Table 1. $\tilde{f}^{i}=\left(\tilde{f}_{1}^{i}, \tilde{f}_{2}^{i}, \ldots, \tilde{f}_{p}^{i}\right)$ is the aggregated vector in category $H_{k},(k=1,2,3,4,5)$ with respect to the alternative $A_{i}$ by the intuitionistic trapezoidal fuzzy weighted average (ITFWA) operator, $\tau=\left(\tau_{1}, \tau_{2}, \ldots, \tau_{p}\right)$ is the position weight of $\tilde{f}^{i}=\left(\tilde{f}_{1}^{i}, \tilde{f}_{2}^{i}, \ldots, \tilde{f}_{p}^{i}\right)$, calculated in the non-priority conditions, here $\tau=(0.3,0.2,0.1,0.2,0.2)^{T}$, and $w_{i}=\left(w_{i 1}, w_{i 2}, w_{i 3}, w_{i 4}, w_{i 5}\right)^{T}$ is the weight vector associated with the category $H_{1}, H_{2}, H_{3}, H_{4}, H_{5}$ with respect to the alternative $A_{i}$, $w_{i k} \in[0,1], i=1,2,3,4, k=1,2,3,4,5 . \sum_{k=1}^{5} w_{i k}=1$. The aim is to select the most suitable can-
didate.

Table 1. Decision-making matrix $\tilde{A}=\left(\tilde{a}_{i j}\right)_{m \times n}$

\begin{tabular}{cccc}
\hline & $C_{11}$ & $C_{12}$ & $C_{13}$ \\
\hline$A_{1}$ & $<[0.3,0.4,0.5,0.6] ; 0.5,0.3>$ & $<[0.7,0.8,0.9,1.0] ; 0.8,0.2>$ & $<[0.4,0.5,0.6,0.7] ; 0.5,0.3>$ \\
\hline$A_{2}$ & $<[0.4,0.5,0.6,0.7] ; 0.5,0.2>$ & $<[0.5,0.6,0.6,0.7] ; 0.5,0.4>$ & $<[0.7,0.8,0.9,1.0] ; 0.5,0.4>$ \\
\hline$A_{3}$ & $<[0.5,0.6,0.6,0.7) ; 0.7,0.2>$ & $<[0.4,0.5,0.6,0.7] ; 0.3,0.2>$ & $<[0.5,0.6,0.6,0.7] ; 0.6,0.2>$ \\
\hline$A_{4}$ & $<[0.6,0.7,0.8,0.9] ; 0.8,0.1>$ & $<[0.5,0.6,0.7,0.8] ; 0.6,0.2>$ & $<[0.4,0.5,0.6,0.7] ; 0.9,0.1>$ \\
\hline
\end{tabular}

Table 1. Decision-making matrix $\tilde{A}=\left(\tilde{a}_{i j}\right)_{m \times n}$ (Continued 2)

\begin{tabular}{cccc}
\hline & $C_{21}$ & $C_{31}$ & $C_{41}$ \\
\hline$A_{1}$ & $<[0.7,0.8,0.9,1.0] ; 0.8,0.2>$ & $<[0.7,0.8,0.9,1.0] ; 0.8,0.2>$ & $<[0.7,0.8,0.9,1.0] ; 0.8,0.2>$ \\
\hline$A_{2}$ & $<[0.6,0.7,0.8,0.9] ; 0.7,0.2>$ & $<[0.5,0.6,0.6,0.7] ; 0.5,0.4>$ & $<[0.6,0.7,0.8,0.9] ; 0.7,0.2>$ \\
\hline$A_{3}$ & $<[0.3,0.4,0.5,0.6] ; 0.7,0.2>$ & $<[0.4,0.5,0.6,0.7] ; 0.3,0.2>$ & $<[0.3,0.4,0.5,0.6] ; 0.7,0.2>$ \\
\hline$A_{4}$ & $<[0.4,0.5,0.6,0.7] ; 0.5,0.3>$ & $<[0.5,0.6,0.7,0.8] ; 0.6,0.2>$ & $<[0.4,0.5,0.6,0.7] ; 0.5,0.3>$ \\
\hline
\end{tabular}

Table 1. Decision-making matrix $\tilde{A}=\left(\tilde{a}_{i j}\right)_{m \times n}$ (Continued 1)

\begin{tabular}{ccc}
\hline & $C_{42}$ & $C_{51}$ \\
\hline$A_{1}$ & $<[0.3,0.4,0.5,0.6] ; 0.5,0.3>$ & $<[0.7,0.8,0.9,1.0] ; 0.8,0.2>$ \\
\hline$A_{2}$ & $<[0.4,0.5,0.6,0.7] ; 0.5,0.2>$ & $<[0.5,0.6,0.6,0.7] ; 0.5,0.4>$ \\
\hline$A_{3}$ & $<[0.5,0.6,0.6,0.7] ; 0.7,0.2>$ & $<[0.4,0.5,0.6,0.7] ; 0.3,0.2>$ \\
\hline$A_{4}$ & $<[0.6,0.7,0.8,0.9] ; 0.8,0.1>$ & $<[0.5,0.6,0.7,0.8] ; 0.6,0.2>$ \\
\hline
\end{tabular}

\subsection{Multi-attribute decision-making method based on the ITFPOWA operator}

Step 1. Integrate the criteria values in 5 class criteria. Then, get the decision-making matrix $\tilde{f}^{i}=\left(\tilde{f}_{1}^{i}, \tilde{f}_{2}^{i}, \ldots, \tilde{f}_{p}^{i}\right)$, respectively:

$$
\tilde{f}=\left[\begin{array}{l}
<[0.14,0.17,0.20,0.23] ; 0.26,0.67><[0.14,0.16,0.18,0.20] ; 0.28,0.72><[0.14,0.16,0.18,0.20] ; 0.28,0.72> \\
<[0.16,0.19,0.21,0.24] ; 0.19,0.71><[0.12,0.14,0.16,0.18] ; 0.21,0.72><[0.10,0.12,0.12,0.14] ; 0.13,0.83> \\
<[0.14,0.17,0.18,0.21] ; 0.22,0.62><[0.06,0.08,0.10,0.12] ; 0.21,0.72><[0.08,0.10,0.12,0.14] ; 0.07,0.72> \\
<[0.15,0.18,0.21,0.24] ; 0.38,0.54><[0.08,0.10,0.12,0.14] ; 0.13,0.79><[0.10,0.12,0.14,0.16] ; 0.17,0.72>
\end{array}\right.
$$




$\left.\begin{array}{l}<[0.10,0.12,0.14,0.16] ; 0.21,0.75><[0.07,0.08,0.09,0.10] ; 0.15,0.85> \\ <[0.10,0.12,0.14,0.16] ; 0.17,0.72><[0.05,0.06,0.06,0.07] ; 0.07,0.91> \\ <[0.08,0.10,0.11,0.13] ; 0.21,0.72><[0.04,0.05,0.06,0.07] ; 0.04,0.85> \\ <[0.10,0.12,0.14,0.16] ; 0.21,0.70><[0.05,0.06,0.07,0.08] ; 0.09,0.85>\end{array}\right]$.

Step 2. Use the ITFPOWA operator to calculate the integrated values $\tilde{f}^{i}$ for every alternative, respectively.

$V$ is a matrix of expectations, where $V_{i k}$ are expectations of $\tilde{f}_{k}^{i}$. We can get

$$
V=\left[\begin{array}{lllll}
0.055 & 0.047 & 0.047 & 0.029 & 0.013 \\
0.048 & 0.037 & 0.018 & 0.029 & 0.005 \\
0.053 & 0.022 & 0.019 & 0.026 & 0.005 \\
0.082 & 0.019 & 0.029 & 0.033 & 0.008
\end{array}\right]
$$

Calculate the associated weighted vector $w_{i}=\left(w_{i 1}, w_{i 2}, \ldots, w_{i p}\right)^{T}$.

Take $A_{1}$ for an example. According to Formulas (24-27) we can get

$$
\begin{aligned}
& T_{1}=1, T_{2}=0.05455, T_{3}=0.00255, T_{4}=0.000119, T_{5}=0.000003 ; \\
& r=(0.94587,0.051596,0.002414,0.000113,0.000003) ; \\
& R=(0.945874,0.99747,0.99988,0.99997,1) ; \\
& w_{1}=(0.94587,0.051596,0.002414,0.000113,0.000003) .
\end{aligned}
$$

Calculate the integrated values $\tilde{f}^{i}$ :

$$
\tilde{f}^{1}=\operatorname{ITFPOWA}\left(A_{1}\right)=<[0.14,0.17,0.20,0.23] ; 0.26,0.67>.
$$

Similarly, we can get

$$
\begin{aligned}
& \tilde{f}^{2}=\operatorname{ITFPOWA}\left(A_{2}\right)=<[0.16,0.19,0.21,0.24] ; 0.19,0.71> \\
& \tilde{f}^{3}=\operatorname{ITFPOWA}\left(A_{3}\right)=<[0.14,0.17,0.18,0.21] ; 0.22,0.62> \\
& \tilde{f}^{4}=\operatorname{ITFPOWA}\left(A_{4}\right)=<[0.14,0.16,0.17,0.20] ; 0.37,0.62>.
\end{aligned}
$$

Step 3. Use the matrix of possibility degree to rank all alternatives.

According to Formula (13), we can build the matrix of the possibility degree:

$$
P=\left[\begin{array}{cccc}
0.5 & 1 & 0.864 & 0 \\
0 & 0.5 & 0 & 0 \\
0.136 & 1 & 0.5 & 0 \\
1 & 1 & 1 & 0.5
\end{array}\right]
$$

Then, we can get the sorting vector $\lambda=(2.364,0.500,1.636,3.500)$.

As $\lambda_{4}>\lambda_{1}>\lambda_{3}>\lambda_{2}$, so $A_{4} \succ A_{1} \succ A_{3} \succ A_{2}$, we can conclude that $A_{4}$ is the best candidate.

In order to verify the validity of the proposed method, we used the weighted geometric aggregation operator as suggested by Liu and Jin (2012a) to solve this decision-making 
problem, and we obtained the ranking result $A_{4} \succ A_{1} \succ A_{3} \succ A_{2}$. Obviously, the same ranking results have been produced by two methods. However, the newly proposed decision-making method can consider the prioritised relationship among the attributes, and in the method proposed by Liu and Jin (2012a), the attribute values were considered to have an equal status.

\section{Conclusions}

In real decision-making, there are many multiple attribute decision-making (MADM) problems, in which there exists the prioritised relationship among decision-making attributes. At the same time, the intuitionistic trapezoidal fuzzy numbers make it easier to express the fuzzy information. So, the research on the prioritised fuzzy MADM method based on the intuitionistic trapezoidal fuzzy numbers has broad application prospects. In addition, the traditional POWA operator is generally suitable for aggregating the information taking the form of numerical values, and yet it fails in adapting to intuitionistic trapezoidal fuzzy numbers. Therefore, in this paper, with respect to MADM problems whose attributes have the prioritised relations and the values of attributes take the form of intuitionistic trapezoidal fuzzy numbers, we combined POWA operator with the intuitionistic trapezoidal fuzzy numbers, and have developed an intuitionistic trapezoidal fuzzy prioritised ordered weighted average (ITFPOWA) operator. Some properties of the operator have also been analysed. Furthermore, based on the above operator, we have proposed an approach to decision-making problems, in which the attribute values were intuitionistic trapezoidal fuzzy numbers. The prominent characteristic of the developed approach is that they can take the prioritised relationship between attributes into account. In the end, an illustrative example has been given to show the steps of the proposed method and to indicate its practicality and validity.

Because intuitionistic trapezoidal fuzzy numbers are more suitable to be used for depicting uncertain or fuzzy information, it has been widely used in real decision-making, such as science and technology project review, blind paper review and so on. At the same time, as the aforementioned method can consider the prioritised relationship among attributes, it is more scientific to do decision-making based on them. In the future, we will keep on studying the extension and applications of the established operators to other domains.

\section{Acknowledgments}

This paper has been supported by the National Natural Science Foundation of China (Nos. 71471172 and 71271124), the Special Funds of Taishan Scholars Project of Shandong Province, National Soft Science Project of China (2014GXQ4D192), the Humanities and Social Sciences Research Project of Ministry of Education of China (No. 13YJC630104), Shandong Provincial Social Science Planning Project (No. 15BGLJ06), and the Natural Science Foundation of Shandong Province (No. ZR2011FM036). 


\section{References}

Atanassov, K. T. 1986. Intuitionistic fuzzy sets, Fuzzy Sets and Systems 20(1): 87-96. http://dx.doi.org/10.1016/S0165-0114(86)80034-3

Atanassov, K. T. 1994. Operators over interval-valued intuitionistic fuzzy sets, Fuzzy Sets and Systems 64(2): 159-174. http://dx.doi.org/10.1016/0165-0114(94)90331-X

Atanassov, K. T.; Gargov, G. 1989. Interval-valued intuitionistic fuzzy sets, Fuzzy Sets and Systems 31(3): 343-349. http://dx.doi.org/10.1016/0165-0114(89)90205-4

Chen, Y. B.; Liu, P. D. 2014. Multi-attribute decision-making approach based on intuitionistic trapezoidal fuzzy general Heronian OWA operator, Journal of Intelligent \& Fuzzy Systems 27(3): 1381-1392.

Guo, T. T.; Zhang, S. H.; Wei, C. P.; Wang, X. J. 2011. Method based on POWC-OWA operator for interval number multi-attribute information aggregation, Journal of Qufu Normal University 37(1): $19-23$.

Liao, H. C.; Xu, Z. S.; Zeng, X. J.; Merigó, J. M. 2015. Framework of group decision making with intuitionistic fuzzy preference information, IEEE Transactions on Fuzzy Systems 23(4): 1211-1227. http://dx.doi.org/10.1109/TFUZZ.2014.2348013

Lin, R.; Zhao, X.; Wei, G. W. 2013. Fuzzy number intuitionistic fuzzy prioritized operators and their application to multiple attribute decision making, Journal of Intelligent and Fuzzy Systems 24(4): 879-888.

Liu, P. D. 2014. Some Hamacher aggregation operators based on the interval-valued intuitionistic fuzzy numbers and their application to group decision making, IEEE Transactions on Fuzzy Systems 22(1): 83-97. http://dx.doi.org/10.1109/TFUZZ.2013.2248736

Liu, P. D.; Jin, F. 2012a. A multi-attribute group decision-making method based on weighted geometric aggregation operators of interval-valued trapezoidal fuzzy numbers, Applied Mathematical Modelling 36(6): 2498-2509. http://dx.doi.org/10.1016/j.apm.2011.09.006

Liu, P. D.; Jin, F. 2012b. Methods for aggregating intuitionistic uncertain linguistic variables and their application to group decision making, Information Sciences 205: 58-71. http://dx.doi.org/10.1016/j.ins.2012.04.014

Liu, P. D.; Jin, F. 2012c. The trapezoid fuzzy linguistic Bonferroni mean operators and their application to multiple attribute decision making, Scientia Iranica 19(6): 1947-1959. http://dx.doi.org/10.1016/j.scient.2012.06.028

Liu, P. D.; Liu, Y. 2014. An approach to multiple attribute group decision making based on intuitionistic trapezoidal fuzzy power generalized aggregation operator, International Journal of Computational Intelligence Systems 7(2): 291-304. http://dx.doi.org/10.1080/18756891.2013.862357

Liu, P. D.; Liu, Z. M.; Zhang, X. 2014. Some intuitionistic uncertain linguistic Heronian mean operators and their application to group decision making, Applied Mathematics and Computation 230: 570-586. http://dx.doi.org/10.1016/j.amc.2013.12.133

Liu, P. D.; Wang, Y. M. 2014. Multiple attribute group decision making methods based on intuitionistic linguistic power generalized aggregation operators, Applied Soft Computing 17: 90-104. http://dx.doi.org/10.1016/j.asoc.2013.12.010

Liu, P. D.; Yu, X. C. 2013. Density aggregation operators based on the intuitionistic trapezoidal fuzzy numbers for multiple attribute decision making, Technological and Economic Development of Economy 19(supplement 1): S454-S470. http://dx.doi.org/10.3846/20294913.2013.881436

Liu, P. D.; Yu, X. C. 2014. 2-dimension uncertain linguistic power generalized weighted aggregation operator and its application for multiple attribute group decision making, Knowledge-Based Systems 57(1): 69-80. http://dx.doi.org/10.1016/j.knosys.2013.12.009

Merigó, J. M.; Gil-Lafuente, A. M.; Yager, R. R. 2015. An overview of fuzzy research with bibliometric indicators, Applied Soft Computing 27: 420-433. http://dx.doi.org/10.1016/j.asoc.2014.10.035 
O'Hagan, M. 1990. Using maximum entropy-ordered weighted average to construct a fuzzy neuron, Proceedings of the 24th Annual IEEE Asilomar Conference on Signals, Systems and Computers, 1990, Pacific Grove, CA, 618-623.

Shu, M. H.; Cheng, C. H.; Chang, J. R. 2006. Using intuitionistic fuzzy sets for fault-tree analysis on printed circuit board assembly, Microelectronics Reliability 46(12): 2139-2148. http://dx.doi.org/10.1016/j.microrel.2006.01.007

Wan, S. P.; Dong, J. Y. 2010. Method of intuitionistic trapezoidal fuzzy number for multi-attribute group decision, Control and Decision 25(5): 773-776.

Wang, J. Q. 2008. Overview on fuzzy multi-criteria decision-making approach, Control and Decision 23(6): 601-606.

Wang, J. Q.; Han, Z. Q.; Zhang, H. Y. 2014. Multi-criteria group decision-making method based on intuitionistic interval fuzzy information, Group Decision and Negotiation 23(4): 715-733. http://dx.doi.org/10.1007/s10726-012-9316-4

Wang, J. Q.; Nie, R. R.; Zhang, H. Y.; Chen, X. H. 2013a. New operators on triangular intuitionistic fuzzy numbers and their applications in system fault analysis, Information Sciences 251: 79-95. http://dx.doi.org/10.1016/j.ins.2013.06.033

Wang, J. Q.; Nie, R. R.; Zhang, H. Y.; Chen, X. H. 2013b. Intuitionistic fuzzy multi-criteria decisionmaking method based on evidential reasoning, Applied Soft Computing 13(4): 1823-1831. http://dx.doi.org/10.1016/j.asoc.2012.12.019

Wang, J. Q.; Zhang, Z. 2008. Programming multi-criteria decision-making method based on intuitionistic fuzzy number with incomplete certain information, Control and Decision 23(10): 1145-1148.

Wang, Y.; Xu, Z. S. 2008. A new method of giving OWA weights, Mathematics in Practice and Theory 38(3): 51-61.

Wei, G. W. 2012. Hesitant Fuzzy prioritized operators and their application to multiple attribute group decision making, Knowledge-Based Systems 31: 176-182.

http://dx.doi.org/10.1016/j.knosys.2012.03.011

Wei, G. W. 2010. Some arithmetic aggregation operators with intuitionistic trapezoidal fuzzy numbers and their application to group decision making, Journal of Computers 5(3): 345-351. http://dx.doi.org/10.4304/jcp.5.3.345-351

Wei, G. W.; Zhao, X.; Wang, H. 2012. An approach to multiple attribute group decision making with interval intuitionistic trapezoidal fuzzy information, Technological and Economic Development of Economy 18(2): 317-330. http://dx.doi.org/10.3846/20294913.2012.676995

Xu, Y. J.; Sun, T.; Li, D. F. 2011. Intuitionistic trapezoidal fuzzy prioritized OWA operator and the application in the multi-criteria decision-making, Control and Decision 26(1): 129-132.

Xu, Y. J.; Wang, H. M.; Merigó, J. M. 2014. Intuitionistic Einstein fuzzy Choquet integral operators for multiple attribute decision making, Technological and Economic Development of Economy 20(2): 227-253. http://dx.doi.org/10.3846/20294913.2014.913273

Yager, R. R. 2004. Modeling prioritized multicriteria decision making, IEEE Transactions on Systems, Man, and Cybernetics, Part B: Cybernetics 34(6): 2396-2404. http://dx.doi.org/10.1109/TSMCB.2004.837348

Yager, R. R. 2008. Prioritized aggregated operators, International Journal of Approximate Reasoning 48(1): 263-274. http://dx.doi.org/10.1016/j.ijar.2007.08.009

Yager, R. R. 2009. Prioritized OWA aggregated, Fuzzy Optimization Decision Making 10(8): 245-262. http://dx.doi.org/10.1007/s10700-009-9063-4

Yan, H. B.; Huynh, V. N.; Nakamori, Y.; Murai, T. 2011. On prioritized weighted aggregation in multicriteria decision making, Expert Systems with Applications 38(1): 812-823.

http://dx.doi.org/10.1016/j.eswa.2010.07.039 
Yu, D.; Shi, S. 2015. Researching the development of Atanassov intuitionistic fuzzy set: Using a citation network analysis, Applied Soft Computing 32: 189-198. http://dx.doi.org/10.1016/j.asoc.2015.03.027

Zadeh, I. A. 1965. Fuzzy sets, Information and Control 8(3): 338-353. http://dx.doi.org/10.1016/S0019-9958(65)90241-X

Zeng, S. Z; Wang, Q. F.; Merigó, J. M.; Pan, T. J. 2014. Induced intuitionistic fuzzy ordered weighted averaging: Weighted average operator and its application to business decision-making, Computer Science \& Information Systems 11(4): 839-857.

Zhang, X.; Jin, F.; Liu, P. D. 2013. A grey relational projection method of multi-attribute decision making based on intuitionistic trapezoidal fuzzy number with weight information unknown, Applied Mathematical Modelling 37(5): 3467-3477. http://dx.doi.org/10.1016/j.apm.2012.08.012

Zhang, X.; Liu, P. D. 2010. Method for aggregating triangular intuitionistic fuzzy information and its application to decision making, Technological and Economic Development of Economy 16(2): 280-290. http://dx.doi.org/10.3846/tede.2010.18

Zhao, X.; Lin, R.; Wei, G. W. 2013. Fuzzy prioritized operators and their application to multiple attribute group decision making, Applied Mathematical Modelling 37(7): 4759-4770. http://dx.doi.org/10.1016/j.apm.2012.09.048

Peide LIU is Doctor and Professor at the School of Management Science and Engineering at Shandong University of Finance and Economics, and assistant director of the Enterprise's Electronic-commerce Engineering Research Center of Shandong. He has authored or co-authored more than 110 publications. His research interests include aggregation operators, fuzzy logic, fuzzy decision-making, and their applications.

Ye LI has Master's Degree received from the University of International Relations in International politics in 2008. Now she is General Manager of Beijing Engineering Research Center of Green Building Technology, and is studying for her doctorate in engineering and project management at Beijing Jiaotong University. She has authored or co-authored 6 publications. Research interests: construction supply chain management, construction technology and organisation, construction energy saving and construction engineering management, multiple criteria decision-making.

Jurgita ANTUVHEVIČIENĖ is Doctor and Professor at the Department of Construction Technology and Management, Vilnius Gediminas Technical University, Vilnius, Lithuania. She is a member of EURO Working Groups Multicriteria Decision Aiding and OR in Sustainable Development and Civil Engineering, Editorial Board member of three international research journals. Her research interests include sustainable development, construction business management and investment, multiple criteria analysis, decision-making theories and decision-support systems. 